\title{
Mixed host organic light-emitting devices with low driving voltage and long lifetime
}

\author{
Jiun-Haw Lee ${ }^{\text {a) }}$ and Chih-I. Wu \\ Graduate Institute of Electro-Optical Engineering and Department of Electrical Engineering, National \\ Taiwan University, No. 1, Sec. 4, Roosevelt Road, Taipei, Taiwan, Republic of China \\ Shun-Wei Liu \\ Institute of Chemistry, Academia Sinica, Taipei, Taiwan, Republic of China \\ Ching-An Huang \\ Department of Mechanical Engineering, Chang-Gung University, Tao-Yuan, Taiwan, Republic of China \\ Yih Chang \\ RiTdisplay Corporation, Hsin-Chu Industrial Park, Taiwan, Republic of China
}

(Received 6 July 2004; accepted 31 January 2005; published online 3 March 2005)

\begin{abstract}
In this letter, we present and analyze the device performance of the mixed host $(\mathrm{MH})$ organic light-emitting devices (OLEDs). The host of the emitting layer (EML) material in this device consists of a hole transport layer (HTL) and an electron transport layer (ETL) fabricated by coevaporation. The bipolar transport characteristic of the MH layer helps to reduce the driving voltage. Device lifetime is increased due to the elimination of the sharp boundary of the HTL/EML interface. Combining the MH structure with a high mobility electron ETL material, bis(10-hydroxybenzo[ $h$ ]qinolinato)beryllium, the OLED has shown a brightness of $27600 \mathrm{~cd} / \mathrm{m}^{2}$ at a driving voltage of $5 \mathrm{~V}$, and a lifetime four times longer than that of a conventional OLED. (C) 2005 American Institute of Physics. [DOI: 10.1063/1.1879093]
\end{abstract}

Organic light-emitting devices (OLEDs) have attracted much attention owing to their advantages of low-power consumption, high brightness, high contrast, and low cost. ${ }^{1,2}$ The structure of a typical OLED is similar to a single heterojunction (HJ) semiconductor light-emitting diode (LED). The HJ structure of a hole transport layer (HTL) and an emitting transport layer (EML) interface confines carriers. However, the carriers piling up would ionize the nearby molecules and degrade the organic material. Since the width of this region is usually very narrow (about $10 \mathrm{~nm}$ ), the generated photons and heat induce a strong photochemical reaction within the small region and reduce the device lifetime. ${ }^{3}$ To improve, we coevaporate the HTL and the electron-transport layer (ETL) organic material and use it as the host of the EML. This mixed host (MH) structure blurs the HTL/EML interface. ${ }^{4,5}$ The blurred interface reduced the carrier accumulation and broadened the emission zone, and hence elongated the operation lifetime. In our MH-OLED devices, the operation lifetime was extended by a factor of 4 as compared to the conventional HJ OLED. Furthermore, the bipolar behavior of the mixed material also helps to increase the carrier mobility of the EML and decrease the driving voltage. ${ }^{6}$ However, current efficiency, which can be derived from the slope of brightness versus current density curves (in terms of $\mathrm{cd} / \mathrm{A}$ ), of a MH-OLED device is usually lower than that of a conventional device.

We have also studied the dependence between operation lifetime and the thickness of MH-EML. The optimized EML thickness in MH devices is $20 \mathrm{~nm}$ that also exhibits the best power efficiency. With the increase of mixing ratio of HTL, a blueshift in emission spectrum was observed, which may come from the solid-state solvation effect, as will be de-

\footnotetext{
${ }^{a)}$ Electronic mail: jhlee@cc.ee.ntu.edu.tw
}

scribed later. ${ }^{7}$ Since the driving voltage is lower and the operation lifetime is longer, the MH-OLED can be used in the devices operated with high peak brightness, such as passive matrix displays and flashlights.

In this study, bis(10-hydroxybenzo[h]qinolinato) beryllium $\left(\mathrm{Bebq}_{2}\right)$, is used as an ETL to reduce the driving voltage. The electron mobility of Bebq2 is one order of magnitude higher than that of the typical ETL material, tris-(8hydroxyquinoline) aluminum $\left(\mathrm{Alq}_{3}\right)$, determined by the time-of-flight measurement. ${ }^{8,9}$ Although the HTL material we used, N,N'-diphenyl-N,N'-bis(1-napthyl)-1,1'-biphenyl4,4'-diamine (NPB), still exhibits a hole mobility of one order of magnitude higher than that of the electron mobility of $\mathrm{Bebq}_{2}$, the device shows more balanced carrier transport characteristics and a lower driving voltage than those of when Alq3 is used as the ETL material.

In all of our samples, we used NPB as the HTL material, 9-Benzothiazol-2-yl-1,1,6,6-tetramethyl-2,3,5,6tetrahydro-1H,4H-11-oxa-3a-aza-benzo anthracene-10-one $(\mathrm{C} 545 \mathrm{~T})$ as the green dopant material, and $\mathrm{Bebq}_{2}$ as the ETL material. Table I illustrates the device structures used in the experiments. The dopant concentration of C545T in EML was $1.25 \%$ in mole fraction. $\mathrm{LiF}$ of $1.2 \mathrm{~nm}$ was used as the electron injection layer, and $\mathrm{Al}$ of a thickness of $100 \mathrm{~nm}$ was used as the cathode. After organic and metal depositions, devices were encapsulated in the glove box with $\mathrm{O}_{2}$ and $\mathrm{H}_{2} \mathrm{O}$ concentrations below $1 \mathrm{ppm}$. Desiccant composed of calcium oxide powder was used to absorb the residue water and oxygen in the encapsulated device. Lifetime was measured while the OLED was driven by constant dc current to emit with the initial luminance of $10000 \mathrm{~cd} / \mathrm{m}^{2}$.

Figure 1(a) shows the current density-voltage, or $J-V$, characteristics of Devices A to F in log-log scale. As shown in Fig. 1(a), the $J-V$ curves can be divided into two groups, 
TABLE I. Layer structures of the OLED devices.

\begin{tabular}{cccccc}
\hline \hline Device & $\begin{array}{c}\text { HTL }(\mathrm{nm}) \\
\text { NPB }\end{array}$ & $\begin{array}{c}\text { HJ (nm) } \\
\text { Bebq2 }\end{array}$ & $\begin{array}{c}\text { MH (nm) } \\
\text { NPB:Bebq2 }\end{array}$ & $\begin{array}{c}\text { Dopant } \\
\text { C545T }\end{array}$ & $\begin{array}{c}\text { ETL (nm) } \\
\text { Bedq2 }\end{array}$ \\
\hline A & 45 & 10 & & & 45 \\
B & 40 & 20 & & & 40 \\
C & 35 & 30 & & & 35 \\
D & 45 & & $10(1: 1)$ & $1.25 \%$ & 45 \\
E & 40 & & $20(1: 1)$ & & 40 \\
F & 35 & & $30(1: 1)$ & & 35 \\
G & 40 & & $20(1: 7)$ & & 40 \\
H & 40 & & $20(1: 7)$ & & 40 \\
\hline \hline
\end{tabular}

i.e., $\mathrm{MH}$ and $\mathrm{HJ}$ devices. $\mathrm{MH}$ devices always have higher current density than HJ devices due to the bipolar characteristics of the MH layers. In a bipolar EML, space charges of electrons and holes recombine to produce double-carrier current which is significantly higher than the single-carrier current. ${ }^{10}$ Compared the $J-V$ characteristics at the same EML thickness, taking Devices $\mathrm{B}$ and $\mathrm{E}$ as an example, the driving voltage is $1.8 \mathrm{~V}$ less in $\mathrm{MH}$ devices as compared to that of HJ devices. The results imply the superior transport capability of the MH layer. With the increase of EML thickness in both $\mathrm{HJ}$ and $\mathrm{MH}$ devices, the driving voltage of an OLED is increased since the dopants, C545T, act as traps to impede the charge transport in organic material. In addition, Device $\mathrm{C}$ exhibits the lowest current density under the same driving voltage. However, it still achieves a high current density of
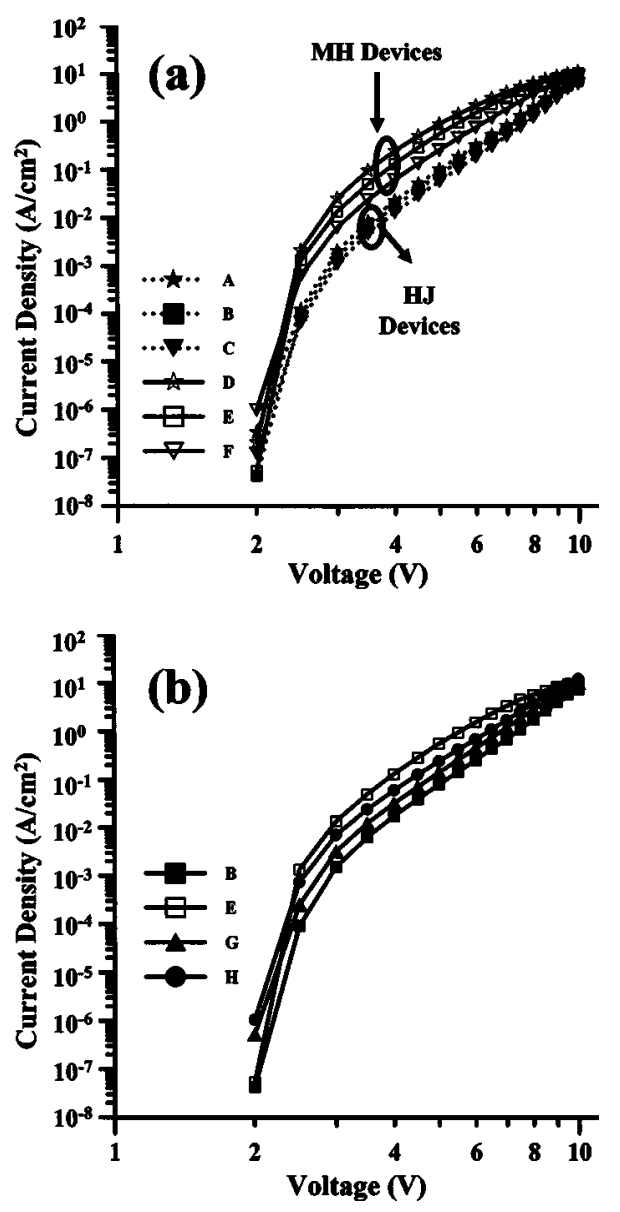

FIG. 1. (a) Current density vs voltage curves of HJ and MH devices with different EML thicknesses. (b) Current density vs voltage curves of $\mathrm{MH}$

devices with different mixing ratios.
Downloaded 17 Feb 2009 to 140.112 .113 .225 . Redistribution subject to AIP license or copyright; see http://apl.aip.org/apl/copyright.jsp

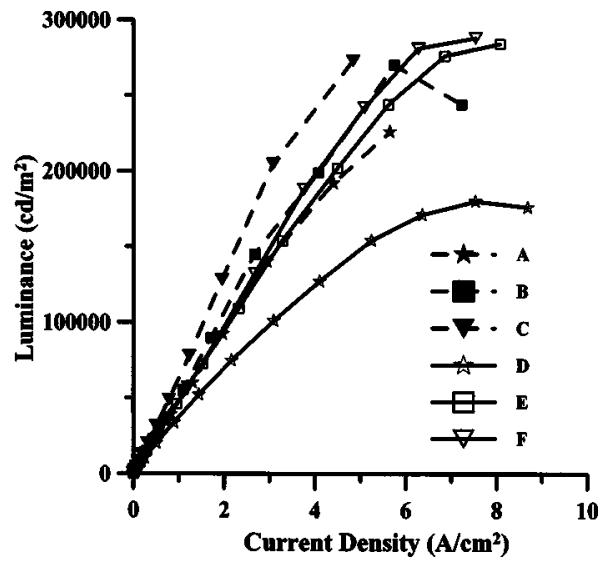

FIG. 2. Luminance vs current density of different devices.

$105 \mathrm{~mA} / \mathrm{cm}^{2}$ at only $5.5 \mathrm{~V}$ that is attributed to the use of high electron mobility ETL. Figure $1(\mathrm{~b})$ shows the $J-V$ characteristics of devices with different mixing ratio. The NPB:Bebq2 ratio is $0: 1,1: 7,1: 1$, and $7: 1$ for Devices $B, G$, $\mathrm{E}$, and $\mathrm{H}$, respectively. It is interesting to note that the current density at the same voltage, for example, $5 \mathrm{~V}$, increases from $79.7,141$, to 553 , and then decreases to $239 \mathrm{~mA} / \mathrm{cm}^{2}$ with increasing mixing ratio. This is explained as follows. When the mixing ratio of NPB is small, the hole mobility of the MH-EML increases with the increase of mixing ratio, which leads to bipolar charge transport. When the mixing ratio is close to $1: 1$, the electron-hole balance is achieved in the EML and the current density reaches maximum. With a further increase of the NPB ratio, the EML is more like holetransport rather than electron-transport material, and consequently, the driving voltage is increased.

Figure 2 shows the dependence of the luminance and the injection current density. Maximum brightness reaches $273000 \mathrm{~cd} / \mathrm{m}^{2}$ and $270000 \mathrm{~cd} / \mathrm{m}^{2}$ for HJ Devicses B and $\mathrm{C}$, respectively. When the driving current is larger than $6 \mathrm{~A} / \mathrm{cm}^{2}$, the brightness begins to decrease. However, in $\mathrm{MH}$ cases, when the current density is over $7 \mathrm{~A} / \mathrm{cm}^{2}$, Devices $\mathrm{E}$ and $F$ reach their maximum brightness of $284000 \mathrm{~cd} / \mathrm{m}^{2}$ and $288000 \mathrm{~cd} / \mathrm{m}^{2}$, respectively, indicating that the $\mathrm{MH}$ devices are more suitable to operate with high driving current. Furthermore, in Devices A and D, the EML is too narrow to have good charge transfer and the $\mathrm{MH}$ device with the thinnest EML (Device D) has the lowest current efficiency. We can see that the absence of a sharp interface in the device reduces the carrier confinement and decreases the current efficiency. In addition, the current efficiency of Device $\mathrm{C}$ is the highest among all of the samples because its HJ structure has the thickest EML.

Figure 3(a) shows the spectra of $\mathrm{MH}$ and $\mathrm{HJ}$ devices with different EML thicknesses. In both the MH and HJ devices, the peaks of the spectra have a redshift of about $2 \mathrm{~nm}$ due to optical interferences as the emitting layer increases. ${ }^{11}$ We can also see that there is a clear blueshift trend from HJ to $\mathrm{MH}$ spectra. Figure 3(b) shows the mixing-ratio dependence of the spectra. When the mixing ratio increases from $0: 1$ to $1: 7$, the spectra are nearly identical with their peak at $522 \mathrm{~nm}$. When the ratio increases from 1:1 to 7:1, the peaks have blueshifts from 516 to $512 \mathrm{~nm}$. The fluorescence spectrum of the device with the polar molecules, C545T, doped into the less polar molecular host, $\mathrm{Bebq}_{2}$, exhibits a redshift due to the formation of ordered polar domains. This is called o AIP license or copyright; see http://apl.aip.org/apl/copyright.jsp 

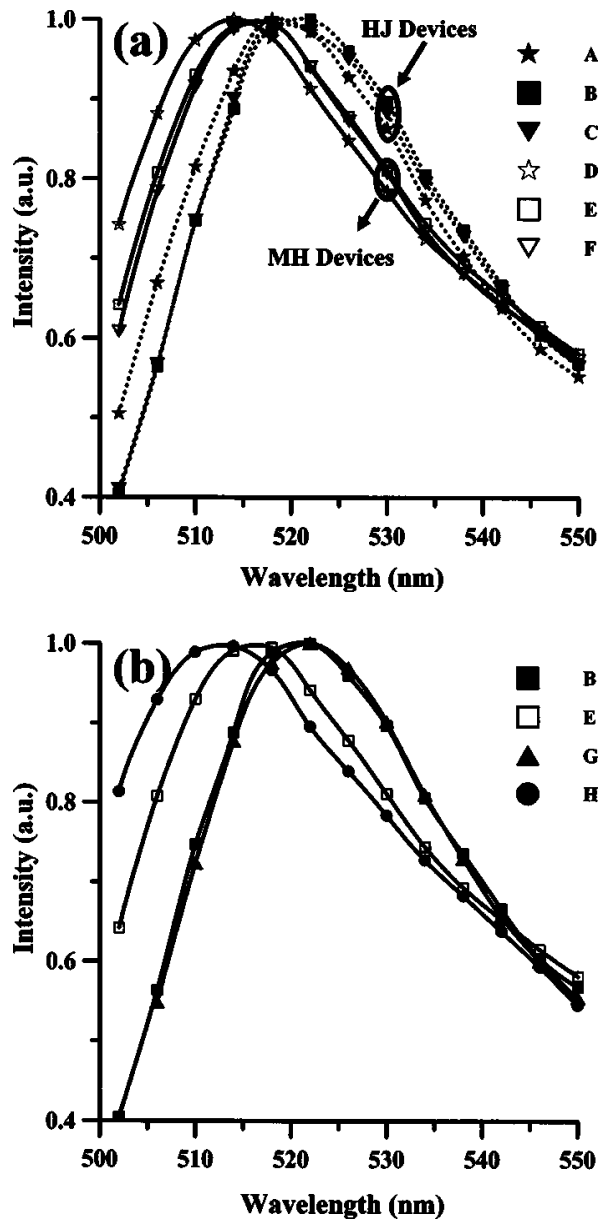

FIG. 3. (a) Spectra of HJ and MH devices with different EML thicknesses. (b) Spectra of MH devices with different mixing ratios.

the solid-state solvation effect. ${ }^{7}$ The magnitude of the spectrum shifts is determined by the domain density in the thin film. The mix of NPB with the host matrix effectively decreases the amount of the domains and blueshifts in the spectrum.

As shown in Fig. 4, because the MH technique reduces the sharp heterojunction interface between HTL and EML and decreases the piling up of the carriers near the interface, it not only reduces the driving voltage but also increases the

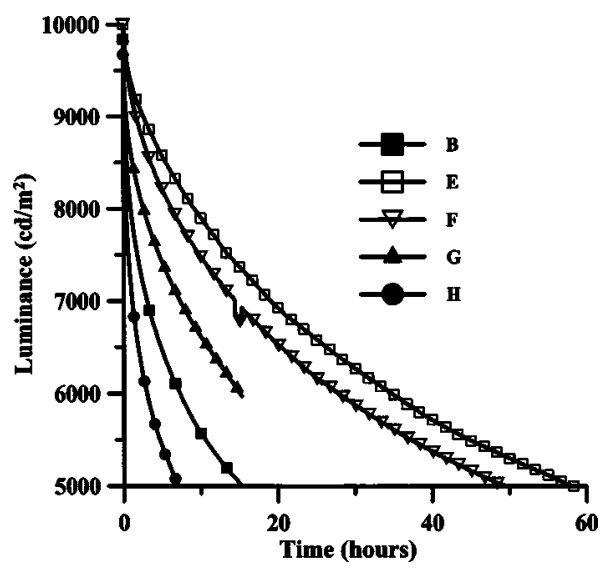

FIG. 4. Accelerated operation lifetime measurement among Devices B, E, F, $\mathrm{G}$, and $\mathrm{H}$. device lifetime. With the initial brightness of $10000 \mathrm{~cd} / \mathrm{m}^{2}$, Device E exhibits a half-lifetime of $58.3 \mathrm{~h}$, nearly four times longer than that of the conventional HJ device (Device B). Other findings are: (a) The shorter lifetime of Device $\mathrm{F}$ as compared to Device E may be due to the lower-power efficiency at this brightness. The thicker MH layer may not give rise to higher lifetime and better device performance, hence device optimization is necessary. (b) When the mixing ratio increases to 7:1 in Device $\mathrm{H}$, the lifetime decreases and is shorter than that of the HJ devices. In this case, the NPB mixing ratio is pretty high in the $\mathrm{MH}$ layer and leads to more holes than electrons in this region. The excessive holes may easily form unstable cations in the MH-EML which cause organic material degradation and luminance decay as observed in the conventional ETL material, Alq3. ${ }^{3}$ However, in our case, we use a different ETL material, Bebq2 and therefore we need more experimental data to support this argument. Furthermore, we can see that, in Fig. 4, lifetimes in our OLEDs are several tens of hours when the initial brightness is $10000 \mathrm{~cd} / \mathrm{m}^{2}$. This implies that the lifetime should be around thousands of hours if the initial brightness is reduced to $200 \mathrm{~cd} / \mathrm{m}^{2}{ }^{12}$ It is relatively low as compared to than that of a typical green OLED device. In our devices, we use $\mathrm{Bebq}_{2}$ as the host material of the EML which yields a lower current efficiency than a conventional device using $\mathrm{Alq}_{3}$. The lower current efficiency means lower-energy transfer from electrical carriers to optical photons and higher heat generated. The excessive heat produced deteriorates the device and limits its lifetime.

In summary, we have shown high brightness and long lifetime in MH-OLEDs using high mobility ETL materials. The MH technique has effectively reduced the driving voltage via its bipolar transport characteristics. We have made MH-OLEDs of long lifetime which we attribute to the HTL/ EML interface blurring. A four-time increase of lifetime has been shown in the optimized MH OLEDs.

This work was supported by the National Science Council, the Republic of China, under Grant Nos. NSC 92-2218E-002-050 and 93-2120-M-002-013. The authors thank Professor Hua-Kuang Liu and Professor Man-Kit Leung for valuable discussions.

${ }^{1}$ C. W. Tang and S. A. Vanslyke, Appl. Phys. Lett. 51, 913 (1987).

${ }^{2}$ C. W. Tang and S. A. Vanslyke, J. Appl. Phys. 65, 3610 (1989).

${ }^{3}$ D. Y. Kondakov, J. R. Sandifer, C. W. Tang, and R. H. Young, J. Appl. Phys. 93, 1108 (2003).

${ }^{4}$ V. V. N. R. Kishore, M. P. Patankar, N. Periasamy, and K. L. Narasimhan, Synth. Met. 143, 295 (2004).

${ }^{5}$ D. Ma, C. S. Lee, S. T. Lee, and L. S. Hung, Appl. Phys. Lett. 80, 3641 (2002).

${ }^{6}$ V. E. Choong, S. Shi, J. Curless, C. L. Shieh, H. C. Lee, F. So, J. Shen, and J. Yang, Appl. Phys. Lett. 75, 172 (1999).

${ }^{7}$ M. A. Baldo, Z. G. Soos, and S. R. Forrest, Chem. Phys. Lett. 347, 297 (2001).

${ }^{8}$ G. G. Malliaras, Y. Shen, D. H. Dunlap, H. Murata, and Z. H. Kafafi, Appl. Phys. Lett. 79, 2582 (2001).

${ }^{9}$ S. W. Liu, C. A. Huang, and Y. Chang, The Pacific Rim Conference on Lasers and Electro-Optics, Taipei, Taiwan, 15-19 December (2003).

${ }^{10}$ W. Brütting, S. Berleb, and A. G. Mückl, Org. Electron. 2, 1 (2001).

${ }^{11}$ Y. Fukuda, T. Watanabe, T. Wakimoto, S. Miyaguchi, and M. Tsuchida, Synth. Met. 111, 1 (2000).

${ }^{12}$ S. A. Van Slyke, C. H. Chen, and C. W. Tang, Appl. Phys. Lett. 69, 2160 (1996). 